\title{
STOCHASTIC PERTURBATIONS TO CONSERVATIVE DYNAMICAL SYSTEMS ON THE PLANE. II: RECURRENCY CONDITIONS
}

\author{
G. WOLANSKY
}

\begin{abstract}
We consider a conservative system on the plane, subjected to a perturbation. The above perturbation is composed of a deterministic part and a random (white noise) part. We discuss the conditions under which there exists a unique, finite invariant measure to the perturbed system, and the weak compactness of the above measures for small enough perturbation's parameter.
\end{abstract}

1. Introduction and main results. In Part I of this paper [5] we considered the problem of convergence, as $\varepsilon \rightarrow 0$, of the invariant measures due to the diffusion process generated by

$$
L^{\varepsilon}=\frac{1}{\varepsilon} \bar{\nabla} H \cdot \nabla+Q \cdot \nabla+\Delta
$$

on $\mathbf{R}^{2}$. Here $H$ is a smooth, real function with a finite number of critical points, $\bar{\nabla} \equiv(-\partial / \partial y, \partial / \partial x)$ for $z=(x, y), Q$ is a smooth vector-valued function on $\mathbf{R}^{2}$ and $\Delta$ stands for the Laplacian. In [5] we assumed that the diffusion process generated by (1.1) admits a unique invariant measure, $\mu^{\varepsilon}$, on $\mathbf{R}^{2}$, for all $\varepsilon>0$ small enough. Moreover, the set of measures $\left\{\mu^{\varepsilon}\right\}$ was assumed to be weakly compact (tight). Under the above assumption we proved the strong convergence of $\mu^{\varepsilon}$, as $\varepsilon \rightarrow \infty$ to a finite measure $\mu^{0} . \mu^{0}$ was represented by a density function $\rho^{0}$ which is given explicitly as a function of the local action, $J$, due to the Hamiltonian $H$. By our condition on $H, J$ is a smooth, single-valued function on $\{z ;|z| \geq R\}$ for some $R>0 . J$ is defined by

$$
J(z)=\oint_{\gamma(z)} y d x,
$$

where $\gamma(z)$ is the level curve of $H$ intersecting $z$. On each such level curve we defined the averaging operator

$$
\langle\sigma\rangle_{(z)} \equiv \oint_{\gamma(z)} \frac{\sigma\left(z^{\prime}\right)}{\left|\nabla J\left(z^{\prime}\right)\right|} d \Gamma\left(z^{\prime}\right) .
$$

Consider

$$
\psi(J)=\frac{\langle\nabla J \cdot Q\rangle}{\left\langle|\nabla J|^{2}\right\rangle}, \quad J=J(z) .
$$

Received by the editors July $15,1987$.

1980 Mathematics Subject Classification (1985 Revision). Primary 35B20, 35R60, 60J60.

Key words and phrases. Diffusion process, perturbation, positive recurrency. 
By the above notation $\rho^{0}$ was defined outside the $R$ disc on $\mathbf{R}^{2}$ as

$$
\rho^{0}(J) \equiv C \exp \left(\int^{J} \psi(s) d s\right)
$$

where $C$ is a normalization constant and $\rho^{0}(z)$ is defined by (1.5) upon the substitution $J=J(z)[\mathbf{5}]$.

In the present paper we study the conditions under which the assumptions of existence and weak compactness of $\left\{\mu^{\varepsilon}\right\}$ are satisfied. From (1.5) it seems evident that a necessary condition for the above assumptions is the integrability of $\rho^{0}(z)$. In particular, the integrability of $\rho^{0}$ follows from the assumption

$$
\varlimsup_{s \rightarrow \infty} \psi(s)<0 \text {. }
$$

The sufficiency of (1.6) is a delicate problem (see below). As for now, we assume a much stronger assumption:

AsSUMPTION $\mathrm{A}_{1}$.

1.

$$
\varlimsup_{s \rightarrow \infty}\left\{\sup _{z^{\prime} \in \gamma(s)}\left[\frac{Q \cdot n_{J}}{|\nabla J|}+\frac{\Delta J}{|\nabla J|^{2}}\right]\right\}<0
$$

where $\gamma(s)$ is the level curve of $J=s$ and $n_{J}$ is the outward normal to $\gamma(J)$.

2 .

$$
\varliminf_{s \rightarrow \infty}\left\{\inf _{z \in \gamma(s)}\left[|\nabla J|^{2}\right\}=\infty\right. \text {. }
$$

It is easy to see that Assumption $A_{1}$ is stronger than (1.6) provided $\Delta J$ is uniformly bounded. In fact, by (1.3),

$$
\begin{gathered}
\left\langle|\nabla J|^{2}\right\rangle(s)=\oint_{\gamma(s)}|\nabla J|, \\
\langle Q \cdot \nabla J\rangle(s)=\oint_{\gamma(s)} \frac{Q \cdot \nabla J}{|\nabla J|}=\oint_{\gamma(s)} Q \cdot n_{J} .
\end{gathered}
$$

Thus, Assumption $\mathrm{A}_{1}$ requires the strict inequality at any point on the contour $\gamma(s)$ for $s$ large enough, while (1.6) requires only the inequality of the contour averages. Notice that both (1.6) and Assumption $A_{1}$ ignore the functional dependence of $H$ on $J$ (i.e. the frequency of oscillations on level curves of $H$ due to the unperturbed system $\dot{z}=\bar{\nabla} H(z))$. It is only the behavior of $Q \cdot \nabla n_{J}$ and $\nabla J$ that counts.

THEOREM 1.1. Assume $\mathrm{A}_{1}$ holds. Then, for any $\varepsilon>0$ the process generated by (1.1) is positively recurrent. In particular there exists a finite invariant measure $\mu^{\varepsilon}$, associated with the diffusion process generated by $L^{\varepsilon}$. Moreover, the set $\left\{\mu^{\varepsilon}\right\}_{\varepsilon>0}$ is compact in the weak topology of measures.

Consider now the weaker assumption (1.6). As it stands, it is not likely that (1.6) may replace $A_{1}$ in Theorem 1.1. This is due to the fact that (1.6) contains no information about the frequency of oscillations on a given level curve of $H$, and we cannot expect that $|\nabla J|$ and $Q \cdot n_{J}$ can be replaced by its contour averages if the frequency $\omega(J)(=d H(J) \backslash d J)$ is zero for some values of $J$. Moreover, even if $\omega(J)$ admits a positive lower bound, we may expect that the conclusions of Theorem 1.1 
would fail if $Q \cdot \nabla J$ deviates wildly from its contour average. If $H$ is a harmonic oscillator

$$
H(x, y)=\frac{1}{2} x^{2}+\frac{1}{2} \omega^{2} y^{2}, \quad \omega=\text { const } \neq 0
$$

then we can prove an analog of Theorem 1.1:

THEOREM 1.2. Assume $H$ is given by (1.9) and, in addition, $\exists C>0$

$$
\left|\nabla_{z} Q\right|<C, \quad \forall z \in \mathbf{R}^{2} \text {. }
$$

Then the conclusions of Theorem 1.1 hold provided (1.6) replaces Assumption $\mathrm{A}_{1}$ and $\varepsilon>0$ is small enough.

Condition (1.9) can be relaxed as follows: If $H$ is harmonic, then the associated action is given by $J=H / \omega$. Here $\omega$ is a positive constant so $J$ is a definite quadratic form. Consider a general Hamiltonian $H=H(z)$, for which the associated action satisfies the following generalization of the "harmonic" action:

$$
\begin{gathered}
O(J)=O\left\langle|\nabla J|^{2}\right\rangle=O(|z|)^{2} \quad \text { as } z \rightarrow \infty \\
\left|\nabla_{z} \nabla_{z} J\right|<C .
\end{gathered}
$$

In addition, let the canonical angle $\theta(\{J, \theta\}$ form a canonical pair) satisfy the obvious generalization to the harmonic oscillator case

$$
|z|\left|\nabla_{z} \theta\right|+\left|\Delta_{z} \theta\right| \leq C .
$$

THEOREM $1.2^{\prime}$. Let $H$ be a Hamiltonian function on $\mathbf{R}^{2}$ and assume the associated action-angle variables satisfy (1.11)-(1.13) for $z$ at a certain neighborhood of infinity. Assume, in addition,

$$
C_{2}>d H / d J \equiv \omega(J)>C_{1}>0
$$

and $\bar{\nabla} H$ is uniformly Lipschitz (as a function of $z$ ). Then the conclusion of Theorem 1.1 holds if (1.6) replaces Assumption $\mathrm{A}_{1}$.

Notice the restriction " $\varepsilon$ small enough" in Theorem $1.2\left(2^{\prime}\right)$ compared to "any $\varepsilon>0$ " in Theorem 1.1. Theorem $1.2\left(2^{\prime}\right)$ may be applied to the case of degenerate diffusion as well. In this case, we replace the Laplacian in (1.1) by, say, $\partial^{2} / \partial x^{2}$. The formal expression of $\rho^{0}(1.5)$ is the same, where $\left\langle|\partial J / \partial x|^{2}\right\rangle,\left\langle\partial^{2} J / \partial x^{2}\right\rangle$ replace $\left\langle|\nabla J|^{2}\right\rangle$ and $\langle\Delta J\rangle$, respectively, in (1.4). Here the analog of Assumption $A_{1}$ fails since $\left(\partial^{2} J / \partial x^{2}\right) /|(\partial J / \partial x)|^{2}$ blows up at some point on any level curve of $J$, while (1.6) may be satisfied since $\left\langle|\partial J / \partial x|^{2}\right\rangle$ is always positive.

2. Technical background. In order to show the existence of a finite invariant measure $\mu^{\varepsilon}$, one has to prove the positive recurrency of the process involved [3]. Given a simply connected bounded domain $\Omega_{1}$, let $\tau_{1}^{\varepsilon}$ be the hitting time of $\gamma_{1}=$ $\partial \Omega_{1}$. If $\Omega_{2} \supset \supset \Omega_{1}$ and $\gamma_{2}=\partial \Omega_{2}$, then it is enough to show:

$$
\sup _{x \in \gamma_{2}} E_{x}\left(\tau_{1}^{\varepsilon}\right)<\infty
$$

(see [1]). In the case of Theorem 1.1 we apply some adaptation of the barrier method [1] and use the special structure of $L^{\varepsilon}$. Defining $\gamma_{1}, \gamma_{2}$ as level contours of $J$, we explicitly define a function $U^{0}=U^{0}(J)$ satisfying

$$
L^{\varepsilon} U^{0}(J)<0, \quad \lim _{J \rightarrow \infty} U^{0}(J)=\infty .
$$


The existence of $U^{0}$ as above guarantees the recurrency of the process. To prove the positive recurrency, we construct a second barrier $F=F(J)>0$

$$
L^{\varepsilon}(F) \leq-1 \quad \forall z,\left\{J(z) \geq J_{1}\right\},
$$

where $J(z)=J_{i}$ on $\gamma_{i}, i=1,2$. Then

$$
E_{z}\left(\tau_{1}^{\varepsilon}\right) \leq F\left(J_{2}\right) \quad \forall z \in \gamma_{2}
$$

and the positive recurrency follows from (2.1). Since $F$ is independent of $\varepsilon$, the l.h.s. of (2.1) turns out to be independent of $\varepsilon$ as well. This observation, however, is not sufficient for the weak compactness of the invariant distributions $\left\{\mu^{\varepsilon}\right\}$.

In order to prove the weak compactness, we use a representation of an invariant distriubtion $\mu$ to a positively recurrent diffusion process, due to Khas'minskii [4]. Consider the stopping time $\tau^{\varepsilon}$ :

$$
\tau^{\varepsilon}=\left\{\inf t>0 ; z^{\varepsilon}(t) \in \Omega_{1}, \exists(0 \leq s \leq t) z^{\varepsilon}(s) \in \sim \Omega_{1}\right\} .
$$

Then, if $A$ is a Borel set

$$
\mu^{\varepsilon}(A)=\int_{\gamma_{1}} E_{y}\left(\int_{0}^{\tau^{\varepsilon}} \chi_{A}\left(z^{\varepsilon}(s)\right) d s\right) d \nu^{\varepsilon}(y) / \int_{\gamma_{1}} E_{y}\left(\tau^{\varepsilon}\right) d y
$$

where $\nu^{\varepsilon}$ is a probability measure on $\gamma_{1}$ induced by the diffusion process $\left(\chi_{A}\right.$-the characteristic function of $A$ ). In [5] we used (2.5) to obtain the strong convergence $\mu^{\varepsilon} \rightarrow \mu^{0}$ on $\Omega_{1}$. Here we use the same representation to estimate $\mu^{\varepsilon}$ on the external domain $\sim \Omega_{2}$. Let $\{z ;|z| \geq R\}:=\Omega_{R}^{\infty} \subset \sim \Omega_{2}$ for $R>0$ large enough. By (2.5), $\mu^{\varepsilon}\left(\Omega_{R}^{\infty}\right)$ is estimated by

$$
\mu^{\varepsilon}\left(\Omega_{R}^{\infty}\right) \leq \sup _{z \in \gamma_{1}} E_{z}\left(\int_{0}^{\tau^{\varepsilon}} \chi_{R}^{\infty}\left(z^{\varepsilon}(s)\right) d s\right) / \inf _{z \in \gamma_{1}} E_{z}\left(\tau^{\varepsilon}\right),
$$

where $\chi_{R}^{\infty}$ is the characteristic function of $\Omega_{R}^{\infty}$. Since $\chi_{R}^{\infty}$ is supported in $\sim \Omega_{2}$, we obtain for $z \in \gamma_{1}$

$$
E_{z}\left(\int_{0}^{\tau^{\varepsilon}} \chi_{R}^{\infty}\left(z^{\varepsilon}(s)\right) d s\right)=E_{z}\left(\int_{\tau_{2}^{\varepsilon}}^{\tau^{\varepsilon}} \chi_{R}^{\infty}\left(z^{\varepsilon}(s)\right) d s\right)
$$

where $\tau_{2}^{\varepsilon}$ is the hitting time of $\gamma_{2}$. By the strong Markov property

$$
\begin{aligned}
E_{z}\left(\int_{\tau_{2}^{\varepsilon}}^{\tau^{\varepsilon}} \chi_{R}^{\infty}\left(z^{\varepsilon}(s)\right) d s\right) & =E_{z}\left(E_{z^{\varepsilon}}\left(\tau_{2}^{\varepsilon}\right)\left(\int_{0}^{\tau_{1}^{\varepsilon}} \chi_{R}^{\infty}\left(z^{\varepsilon}(s)\right) d s\right)\right) \\
& \leq \sup _{x \in \gamma_{2}} E_{x}\left(\int_{0}^{\tau_{1}^{\varepsilon}} \chi_{R}^{\infty}\left(z^{\varepsilon}(s)\right) d s\right),
\end{aligned}
$$

where $\tau_{1}^{\varepsilon}$ is, as before, the hitting time of $\gamma_{1}$. From (2.5) and (2.8)

$$
\mu^{\varepsilon}\left(\Omega_{R}^{\infty}\right) \leq C^{-1} \sup _{x \in \gamma_{2}} E_{x}\left(\int_{0}^{\tau_{1}^{\varepsilon}} \chi_{R}^{\infty}\left(z^{\varepsilon}(s)\right) d s\right),
$$

provided $C$ is an $\varepsilon$ independent function satisfying

$$
\inf _{z \in \gamma_{1}} E_{z}\left(\tau^{\varepsilon}\right)>\inf _{x \in \gamma_{2}} E_{x}\left(\tau_{1}^{\varepsilon}\right)>C>0 .
$$


Thus we prove the weak compactness of $\left\{\mu^{\varepsilon}\right\}$ by showing

$$
\lim _{R \rightarrow \infty}\left\{\sup _{x \in \gamma_{2}} E_{x}\left(\int_{0}^{\tau_{1}^{\varepsilon}} \chi_{R}^{\infty}\left(z^{\varepsilon}(s)\right) d s\right)\right\}=0
$$

holds uniformly w.r. to $\varepsilon>0$. In the beginning of $\S 3$ we fill in the details of the proof of Theorem 1.1.

The proof of Theorem 1.2(2') is more complicated. Here we cannot introduce an explicit barrier as in (2.3). However, there exists a function $U^{*}(J)>0$ satisfying

$$
L^{\varepsilon} U^{*}=-1+\xi
$$

where $\langle\xi\rangle_{(J)}=0$ for $J \geq J_{1}$ (see (1.3) for the definition of $\langle\cdot\rangle$ ). In order to prove (2.1) in this case, one has to show

$$
E_{x}\left(\int_{0}^{\tau_{1}^{\varepsilon}} \xi\left(z^{\varepsilon}(s)\right) d s\right) \leq \delta E_{x}\left(\tau_{1}^{\varepsilon}\right)+C<\infty
$$

for $x \in \gamma_{2}, C>0,0<\delta<1$, where $C, \delta$ are independent of the particular choice of $z \in \gamma_{2}$. To prove (2.13), we take advantage of the definition of $\xi$ :

$$
\int_{0}^{T_{z}^{0}} \xi\left(z^{0}(s)\right) d s=0
$$

where $z^{0}(s)$ satisfies the unperturbed (deterministic) system

$$
z^{0}=\bar{\nabla} H\left(z^{0}\right), \quad z^{0}(0)=z
$$

and $T_{z}^{0}=2 \pi / \omega(z)$. (2.14) is just the condition $\langle\xi\rangle \equiv 0$. Splitting $\xi=\tilde{\xi}+(1-\tilde{\xi})$ where $1-\tilde{\xi}$ is compactly supported in $\sim \Omega_{1}$ and $\langle\tilde{\xi}\rangle=0,(2.13)$ is reduced to (see $\S 3)$ :

$$
E_{x}\left(\int_{0}^{\tau_{1}^{\varepsilon}} \tilde{\xi}\left(z^{\varepsilon}(s)\right) d s\right) \leq \delta E_{x}\left(\tau_{1}^{\varepsilon}\right)<\infty
$$

for $\delta<1$ and $x \in \gamma_{2}$, fixed.

Let $x \in \gamma_{2}, \theta(x)=\theta_{0}$. We define the ray $l_{\theta_{0}}^{\eta} \subset \sim \Omega_{1}$ as

$$
l_{\theta_{0}}^{\eta}=\left\{z \in \sim \Omega_{1} ; J(z) \geq J_{1}+\eta, \theta(z)=\theta_{0}\right\},
$$

where $\eta>0$. Define $\tilde{\tau}_{1}^{\varepsilon, 1}$ as the stopping time according to the following rule: The process $z^{\varepsilon}(s)$ starting at $\omega \in l_{\theta_{0}}^{\eta}$ is stopped whenever $z^{\varepsilon}(t)$ hits $l_{\theta_{0}}^{\eta}$ after completing at least one revolution in $\theta$. Let $\tau_{1}^{\varepsilon, 1}=\tilde{\tau}_{1}^{\varepsilon, 1} \wedge \tau_{1}^{\varepsilon}$. Using the strong Markov property, we show (§3) that $\left(2.13^{\prime}\right)$ is reduced to the following

$\left.\begin{array}{l}\text { (a) } \forall w \in l_{\theta_{0}}^{\eta}, \quad E_{w}\left(\tau_{1}^{\varepsilon, 1}\right)<\infty \\ \text { (b) } \forall w \in l_{\theta_{0}}^{\eta}, \quad E_{w}\left(\int_{0}^{\tau^{\varepsilon, 1}} \tilde{\xi}\left(z^{\varepsilon}(s) d s\right)\right) \leq \delta E_{w}\left(\tau_{1}^{\varepsilon, 1}\right)\end{array}\right\}$.

(2.16a) is proved in $\S 3$ by constructing an appropriate barrier function. The proof of (2.16b) includes most of the technical details of this paper, and is given in Appendix B. The object of this proof is to estimate the deviation of the l.h.s. of (2.16b) from that of (2.14), for $\varepsilon$ small. In order to prove those estimates, we need a uniform 
lower bound on $E_{w}\left(\tau_{1}^{\varepsilon, 1}\right) \forall w \in l_{\theta_{0}}^{n}$. This is the reason for the choice $J(\omega) \geq J_{1}+\eta$ in the definition of $l_{\theta_{0}}^{\eta}(2.15)$. In Appendix A we prove certain bounds on $\xi$ which are required for the proof of $(2.16 \mathrm{~b})$. The proof of weak compactness follows along similar lines, using (2.11).

\section{Estimates on the external domain.}

PROOF OF THEOREM 1.1. Let the assumptions of Theorem 1.1 hold. For $J>0$ large enough, define

$$
\gamma_{J}=\left\{z \in \mathbf{R}^{2}, J(z)=J\right\} \subset \mathbf{R}^{2} .
$$

Let $J_{2}>J_{1}$, and consider $\gamma_{i}=\gamma_{J_{i}}, i=1,2$. By Assumption $\mathrm{A}_{1}$ we may define $J_{1}$ large enough for which

$$
\sup _{J \in \gamma_{J}} \frac{\Delta J+Q \cdot \nabla J}{|\nabla J|^{2}}<-\alpha<0
$$

where $J \geq J_{1}$ and $\alpha$ are a positive constant. Given $R>R^{\prime} \geq J_{2}$ we define the domain

$$
\Omega_{R^{\prime}}^{R}=\left\{z ; R^{\prime} \leq J(z) \leq R\right\} .
$$

Let $\tau_{1, R}^{\varepsilon}$ be the escape time from $\Omega_{J_{1}}^{R}$. From the definition of $L^{\varepsilon}$ and by $\bar{\nabla} H \cdot \nabla J \equiv 0$ we obtain

$$
L^{\varepsilon}\left(e^{\alpha J}\right)=|\nabla J|^{2} e^{\alpha J}\left[\alpha^{2}+\alpha \frac{\Delta J+Q \cdot \nabla}{|\nabla J|^{2}}\right]
$$

Hence, by (3.2),

$$
L^{\varepsilon}\left(e^{\alpha J}\right) \leq 0
$$

for $J \geq J_{1}$.

Using the Ito formula and applying expectations on the process starting at $\gamma_{2}$ :

$$
E_{y} \int_{0}^{\tau_{1, R}^{\varepsilon}} L^{\varepsilon}\left(e^{\alpha J}\right) d s=E_{y}\left[\exp \alpha J\left(\tau_{1, R}^{\varepsilon}\right)\right]-e^{\alpha J_{2}} \leq 0
$$

for $y \in \gamma_{2}$. The definition of $\tau_{1, R}^{\varepsilon}$ and (3.6) yield

$$
P_{y}\left\{z^{\varepsilon}\left(\tau_{1, R}^{\varepsilon}\right) \in \gamma_{R}\right\} \leq e^{\alpha\left(J_{2}-R\right)}
$$

for arbitrary $R>J_{2}$. Hence the process is regular and recurrent [1]; $\tau_{\varepsilon}^{1}$, the hitting time of $\gamma^{1}$, is finite a.e. and

$$
\lim _{R \rightarrow \infty} \tau_{1, R}^{\varepsilon}=\tau_{1}^{\varepsilon} \quad \text { in prob. }
$$

In particular, for $R \uparrow \infty \tau_{1, R}^{\varepsilon}$ is monotone and (3.7) holds a.s. Let

$$
\delta(J)=\inf _{z \in \gamma_{J}}|\nabla J|^{2}
$$

and

$$
F(J)=\int_{J_{1}}^{J} e^{\alpha s}\left[\int_{s}^{\infty} \frac{1}{\delta(u)} e^{-\alpha u} d u\right] d s
$$


$F$ is well defined by Assumption $\mathrm{A}_{1}(2)$. Applying $L^{\varepsilon}$ on $F$, using (3.2), we obtain in a similar way to $(3.5)$

$$
L^{\varepsilon}(F) \leq-1 \quad \text { for } J \geq J_{1} .
$$

Given $y \in \gamma_{2}$, the Ito formula yields

$$
\left.E_{y} \tau_{1, R}^{\varepsilon} \leq-E_{y} \int_{0}^{\tau_{1, R}^{\varepsilon}} L^{\varepsilon} F\left(z^{\varepsilon}(s)\right) d s=-\left\{E_{y} F\left(\tau_{1, R}^{\varepsilon}\right)\right)-F\left(J_{2}\right)\right\} \leq F\left(J_{2}\right) .
$$

Using (3.7) and the remark thereafter, we obtain a bound on $E_{y} \tau_{1}^{\varepsilon}$ by (3.10) and the monotone convergence. Hence the process is positively recurrent for any $\varepsilon>0$ [1]. In particular, the process (1.3) admits a finite invariant measure, $\mu^{\varepsilon}$.

Let $\Omega_{R}^{\infty}$ be defined by (3.3). The set $\left\{\mu^{\varepsilon}\right\}, \varepsilon>0$, is weakly compact provided

$$
\lim _{R \rightarrow \infty} \mu^{\varepsilon}\left(\Omega_{R}^{\infty}\right)=0, \quad \text { uniformly in } \varepsilon>0 \text {. }
$$

Let now

$$
F^{R}(J)=\int_{J_{1}}^{J} e^{\alpha s}\left[\int_{s}^{\infty} \frac{\chi_{R}^{\infty}}{\delta(u)} e^{-\alpha u} d u\right] d s
$$

where $\chi_{R}^{\infty}$ is the characteristic function on $[R, \infty)$ and $\alpha$ is as in (3.2). A calculation similar to that of $(3.9)$ yields

$$
L^{\varepsilon} F^{R} \leq-\chi_{R}^{\infty}
$$

Hence, for $y \in \gamma_{2}$

$$
\begin{aligned}
E_{y}\left(\int_{0}^{\tau_{1}^{\varepsilon}} \chi_{R}^{\infty}\left(z^{\varepsilon}(s)\right) d s\right) & \leq-E_{y}\left(\int_{0}^{\tau_{1}^{\varepsilon}} L^{\varepsilon} F^{R}\left(z^{\varepsilon}(s)\right) d s\right) \\
& =F^{R}\left(J_{2}\right)-E_{y}\left(F^{R}\left(z^{\varepsilon}\left(\tau_{1}^{\varepsilon}\right)\right)\right)=F^{R}\left(J_{2}\right)
\end{aligned}
$$

by the Ito formula. The last equality above follows from the definition of $F^{R}$ and $\tau_{1}^{\varepsilon}$. From (3.14) we obtain the $\varepsilon$-independent estimate:

$$
\sup _{x \in \gamma_{2}} E_{x}\left(\int_{0}^{\tau^{\varepsilon}} \chi_{R}^{\infty}\left(Z^{\varepsilon}(s)\right) d s\right) \leq F^{R}\left(J_{2}\right) .
$$

By (3.12) and $\lim _{J \rightarrow \infty} \delta(J)=\infty$ (due to Assumption $\mathrm{A}_{1}$ ): $\lim _{R \rightarrow \infty} F^{R}\left(J_{2}\right)=0$, and (3.11) follows. To complete the proof, we need to provide a lower bound on $E_{x}\left(\tau^{\varepsilon}\right)$. In order to evaluate such a bound, consider $\tau_{1, R}^{\varepsilon}$, the escape time from $\Omega_{J_{1}}^{R}$ for fixed $F>J_{2}$. Choosing $\beta>0$ large enough, we may estimate

$$
L^{\varepsilon}\left(e^{\beta J}\right) \geq C>0
$$

for $J_{1} \leq J \leq R$ (compare (3.4) and (3.5)). $C$ is $\varepsilon$ independent and, for $y \in \gamma_{2}$, $C^{-1} e^{\beta J_{2}}$ is a lower bound on $E_{y}\left(\tau_{1, R}^{\varepsilon}\right)$, independently of $y$ and $\varepsilon$ (compare (3.10) where the opposite inequality, (3.16), replaces (3.9)). A lower estimate on $E_{x}\left(\tau^{\varepsilon}\right)$, $x \in \gamma_{1}$, is obtained, independently of $x$ and $\varepsilon$, by the strong Markov property. This completes the proof of Theorem 1.1.

PROOF OF THEOREM 1.2. By the conditions of the theorem, the process admits a strong solution for any $\varepsilon>0$. In fact, the drift satisfies

$$
|\bar{\nabla} H(z)+\varepsilon Q(z)| \leq K(1+|z|), \quad z \in \mathbf{R}^{2},
$$


which, together with the Lipschitz conditions on $\bar{\nabla} H$ and $Q$, satisfies the conditions of Theorem 2.1, §5 of [3]. In particular, we obtain for every $T>0$,

$$
\lim _{R \rightarrow \infty} P_{y}\left(\tau_{R}^{\varepsilon} \geq T\right)=1
$$

where $\tau_{R}^{\varepsilon}$ is the escape time from a domain $\Omega_{R} \subset \mathbf{R}^{2}, \Omega_{R} \uparrow \mathbf{R}^{2}$. Let

$$
\chi(J)=\int_{J_{1}}^{J} \frac{\langle Q \cdot \nabla J\rangle_{s}}{\left\langle|\nabla J|^{2}\right\rangle_{s}} d s
$$

and define

$$
U^{*}(J)=\int_{J_{1}}^{J} \frac{1}{\left\langle|\nabla J|^{2}\right\rangle_{s}} e^{-\chi(s)} \int_{s}^{\infty} e^{\chi(u)} d u .
$$

Notice that $U^{*}$ is well defined by (1.6).

Considering $U^{*}$ as a function on the phase space via $U(J(z))$, a direct substitution yields

$$
\begin{aligned}
L^{\varepsilon} U^{*} & =|\nabla J|^{2} \frac{d^{2}}{d J^{2}} U^{*}+(\Delta J+Q \cdot \nabla J) \frac{d}{d J} U^{*} \\
& =-\frac{|\nabla J|^{2}}{\left\langle|\nabla J|^{2}\right\rangle}+\left\{-\frac{\langle\Delta J+Q \cdot \nabla J\rangle}{\left\langle|\nabla J|^{2}\right\rangle}+\frac{\Delta J+Q \cdot \nabla J}{\left\langle|\nabla J|^{2}\right\rangle}\right\} e^{-\chi(J)} \int_{J}^{\infty} e^{\chi(s)} d s .
\end{aligned}
$$

In deriving (3.20) we used the identity

$$
\langle\nabla J \cdot V\rangle=\frac{d}{d J}\langle\operatorname{div} V\rangle
$$

for any smooth vector-valued $V$. The above identity follows from the divergence theorem (see also [5]). Hence

$$
\left\langle L^{\varepsilon} U^{*}\right\rangle=-1 .
$$

LEMMA 3.1. Under the assumptions of Theorem 1.2, there exists a constant $C$, independent of $\varepsilon$, where

$$
\left|L^{\varepsilon} U^{*}\right|+|z|\left|\nabla_{z} L^{\varepsilon} U^{*}\right| \leq C, \quad \forall z \in \Omega_{J_{1}}^{\infty} .
$$

We defer the proof of Lemma 3.1 to Appendix A.

Let $\tau_{1}^{\varepsilon}=\tau$. (We suppress the $\varepsilon$ dependence from now on.) In $\Omega_{J_{1}}^{\infty}$, consider the segment $l_{\theta_{0}}^{\eta}$ given by

$$
l_{\theta_{0}}^{\eta}=\left\{J_{1}+\eta \leq J \leq \infty, \theta=\theta_{0}\right\}
$$

where $\eta>0$ is a constant, and $J_{1}+\eta<J_{2}$. Let

$$
\begin{aligned}
& \tilde{\tau}^{n}=\left\{\inf t, z(t) \in l_{\theta_{0}}^{n}, \exists 0=t_{0}<t_{1}<t_{2}<\cdots<t_{n}=t,\right. \\
&\left.z\left(t_{i}\right) \in l_{\theta_{0}}^{n} \text { and }\left|\arg z\left(t_{i}\right)-\arg z\left(t_{i-1}\right)\right| \geq 2 \pi\right\}, \quad 1 \leq i \leq n .
\end{aligned}
$$

Define $\tau^{n}=\tilde{\tau}^{n} \wedge \tau$. The stopping rule of $\tilde{\tau}^{n}$ is as follows: The process is stopped whenever $z(s)$ hits $l_{\theta_{0}}^{n}$ at the $n$th time, and each two successive hittings are separated by at least one revolution of $\pm 2 \pi$.

By regularity of the process (see (3.17)):

$$
\lim _{n \rightarrow \infty} \tau^{n}=\tau \quad \text { a.s. }
$$


Notice that, at this point, $\tau$ may admit an infinite value. In fact, $\tau=\infty$ if the sample path never hits $\gamma_{1}$. Let

$$
\xi=L^{\varepsilon} U^{*}+1 .
$$

Using the Ito formula with $\tau^{n}$ as a stopping time, we obtain $\forall x \in \gamma_{2}$ :

$$
E_{x} \tau^{n}=U^{*}\left(J_{2}\right)-E_{x} U^{*}\left(z\left(\tau^{n}\right)\right)+E_{x} \int_{0}^{\tau^{n}} \xi(z(s)) d s .
$$

Since $U^{*} \geq 0$ on $\Omega_{1}^{\infty},(3.25)$ yields

$$
E_{x}\left(\tau^{n}\right) \leq U^{*}\left(J_{2}\right)+\left|E_{x} \int_{0}^{\tau^{n}} \xi(z(s)) d s\right|, \quad \forall x \in \gamma_{2} .
$$

LEMMA 3.2. Under the assumptions of Theorem 1.2,

$$
E_{y} \tau^{n}<\infty \quad \forall n, y \in l_{\theta_{0}}^{\eta} .
$$

Moreover, there exist positive $\delta_{1}(\eta), \delta_{2}(\varepsilon, \eta)$

$$
\begin{gathered}
\lim _{n \rightarrow 0} \delta_{1}(\eta)=0, \quad \lim _{\varepsilon \rightarrow 0} \delta_{2}(\varepsilon, n)=0 \quad \forall \eta>0, \\
\left|E_{x} \int_{0}^{\tau^{n}} \xi(z(s)) d s\right| \leq \delta_{1}(\eta)+\delta_{2}(\varepsilon, \eta) E_{x}\left(\tau^{n}\right),
\end{gathered}
$$

where $\underline{x}$ is the intersection of $\gamma_{2}$ with $l_{\theta_{0}}^{\eta}$. In addition, $\delta_{1}$ and $\delta_{2}$ are independent of $\theta_{0}$. (See the definition of $l_{\theta_{0}}^{\eta}$.)

From Lemma 3.2 we obtain a global bound on $E_{y} \tau^{n}$, independent of $n$ and $y \in \gamma_{2}$, provided $\varepsilon$ is small enough. In fact, by (3.26) and Lemma 3.2

$$
E_{x} \tau^{n} \leq\left[\delta_{1}(\eta)+U^{*}\left(J_{2}\right)\right]\left(1-\delta_{2}(\varepsilon, \eta)\right)^{-1},
$$

provided $\varepsilon$ is small enough. This leads to the boundedness of $E_{x}\left(\tau^{n}\right), x=l_{\theta_{0}}^{\eta} \cap \gamma_{2}$, independent of $n$, and hence to a bound on $E_{x}(\tau)$ via (3.23). Since the conclusions of Lemma 3.2 are independent of $\theta_{0}$, we obtain a bound on $E_{y}(\tau)$, uniform on $y \in \gamma_{2}$, and the positive recurrency follows via [1]. The weak compactness of the associated invariant measures follows along a similar line. We define $U_{R}^{*}$, equivalently to (3.19), as

$$
U_{R}^{*}(J)=\int_{J_{1}}^{J} \frac{1}{\left\langle|\nabla J|^{2}\right\rangle_{s}} e^{-\chi(s)} \int_{s}^{\infty} 1[u \geq R] e^{\chi(u)} d u .
$$

We then obtain, equivalently to (3.22),

$$
\left\langle L^{\varepsilon} U_{R}^{*}\right\rangle=-1_{[R, \infty)} .
$$

Let

$$
\xi^{R}=L^{\varepsilon} U_{R}^{*}+1_{[R, \infty)} .
$$

Then $\xi^{R}$ satisfies the same estimate as $\xi$ in Lemma 3.1. Following the argument of $(3.23) \rightarrow(3.27)$, we obtain

$$
\sup _{x \in \gamma_{2}} E_{x} \tau_{R} \leq\left(\delta_{1}(\eta)+U_{R}^{*}\left(J_{2}\right)+\delta_{2}(\varepsilon, \eta) \sup _{y \in \gamma_{2}} E_{y} \tau\right)\left(1-\delta_{2}(\varepsilon, \eta)\right)^{-1}
$$


where $\tau_{R}$ is the occupation time of $\Omega_{R}^{\infty}$ :

$$
\tau_{R}=\int_{0}^{\tau} \chi_{R}^{\infty}\left(z^{\varepsilon}(s) d s .\right.
$$

The right-hand side of (3.28) is arbitrarily small by choosing $\eta, \varepsilon$ sufficiently small and $R$ sufficiently large, using the already known bound on $\sup _{y \in \gamma_{2}} E_{x}(\tau)$ and

$$
\lim _{R \rightarrow \infty} U_{R}^{*}\left(J_{2}\right)=0 \text {. }
$$

Thus, the weak compactness of the invariant measures follows by the same argument as in Theorem 1.1. (See (3.10) and the remark thereafter.)

PROOF OF LEMMA 3.2. Let $0 \leq \alpha^{\eta}(J) \leq 1$ be a $C^{\infty}$ function on $\Omega_{J_{1}}^{\infty}$ satisfying

$$
\begin{gathered}
\alpha^{\eta}(J)=1, \quad J \geq J_{1}+5 \eta, \\
0 \leq \alpha^{\eta}(J) \leq \delta / 4 M \quad \text { on } J_{1} \leq J \leq J_{1}+4 \eta, \\
\alpha^{\eta}\left(J_{1}\right)=0,
\end{gathered}
$$

where $M=\sup _{\Omega_{J_{1}}^{\infty}}|\xi|, M<\infty$, by Lemma 3.1. Let

$$
\tilde{\xi}=\alpha^{\eta} \xi
$$

Then, for $y \in \gamma_{2}$

$$
E_{x} \int_{0}^{\tau^{n}} \xi(z(s)) d s=E_{x} \int_{0}^{\tau^{n}} \tilde{\xi}(z(s)) d s+E_{x} \int_{0}^{\tau^{n}}\left(1-\alpha^{\eta}\right) \xi(z(s)) d s .
$$

The second term on the right of (3.30) is estimated by

$$
M E_{x} \int_{0}^{\tau^{n}}\left[1-\alpha^{\eta}(z(s))\right] d s
$$

Define

$$
U^{\eta}(J)=-M \int_{J_{1}}^{J} e^{-D s} \int_{s}^{\infty} \frac{e^{D u}}{\left\langle|\nabla J|^{2}\right\rangle(u)}\left(1-\alpha^{\eta}(u)\right) d u
$$

where

$$
D>\sup _{z \in \Omega_{J_{1}}^{\infty}}\left|\frac{\Delta J+Q \cdot \nabla J}{|\nabla J|^{2}}\right|
$$

and $D<\infty$ by $(1.10)-(1.12)$. $U^{\eta}$ is well defined by definition of $\alpha^{\eta}$. Moreover,

$$
L^{\varepsilon} U^{\eta}=M\left(1-\alpha^{\eta}\right)+\left(Q \cdot \nabla J+\Delta J-D|\nabla J|^{2}\right) \frac{d}{d J} U^{\eta} \geq M\left(1-\alpha^{\eta}\right),
$$

by definition of $D$ and the negativity of $d U^{\eta} / d J$. Hence

$M E_{y} \int_{0}^{\tau^{n}}\left(1-\alpha^{\eta}(z(s))\right) d s \leq E_{y} \int_{0}^{\tau^{n}} L^{\varepsilon} U^{\eta}=E_{y} U^{\eta}\left(z\left(\tau^{n}\right)\right)-U^{\eta}\left(J_{2}\right) \leq-U^{\eta}\left(J_{2}\right)$.

Define now

$$
\delta_{1}(\eta)=-U^{\eta}\left(J_{2}\right) / M
$$

By definition of $\alpha^{\eta}$

$$
\lim _{n \rightarrow \infty} \delta_{1}(\eta)=0
$$


and from (3.31) we obtain the estimate

$$
\left|E_{y} \int_{0}^{\tau^{n}}\left(1-\alpha^{\eta}\right) \xi(z(s)) d s\right| \leq \delta_{1}(\eta)
$$

In order to complete the proof we have to show the existence of $\delta_{2}(\varepsilon, \eta)$ for which

$$
E_{x}\left|\int_{0}^{\tau^{k}} \tilde{\xi}(z(s)) d s\right| \leq \delta_{2}(\varepsilon, \eta) E_{x}\left(\tau^{k}\right), \quad \forall k, x=l_{\theta_{0}}^{\eta} \cap \gamma_{2},
$$

and the boundedness of the right-hand side of (3.32). We proceed by induction on $\mathbf{k}$ as follows:

Assume $E_{x}\left(\tau^{n}\right)<\infty$ and (3.32) holds for $\delta_{2}(\varepsilon, \eta)=\delta, k=n$. Then

$$
E_{x}\left|\int_{0}^{\tau^{n+1}} \tilde{\xi}(z(s)) d s\right| \leq E_{x}\left|\int_{0}^{\tau^{n}} \tilde{\xi}(z(s)) d s\right|+E_{x}\left(E_{z\left(\tau^{n}\right)}\left|\int_{0}^{\tau^{1}} \tilde{\xi}(z(s)) d s\right|\right)
$$

by the strong Markov property. Similarly

$$
E_{x}\left(\tau^{n+1}\right)=E_{x}\left(\tau^{n}\right)+E_{x}\left(E_{z\left(\tau^{n}\right)} \tau^{1}\right) .
$$

By the induction hypothesis for $k=n$, we obtain (3.32) for $k=n+1$, using (3.33), (3.34), provided

$$
E_{y}\left|\int_{0}^{\tau^{1}} \tilde{\xi}(z(s))\right| \leq \delta E_{y}\left(\tau^{1}\right)
$$

holds for each $y \in \operatorname{Range}\left(z\left(\tau^{1}\right)\right)$, where the r.h.s. of (3.35) is bounded. By definition of $\tau^{n}, z\left(\tau^{n}\right) \subset \gamma_{1} \cup l_{\theta_{0}}^{\eta}$. Since $x=\gamma_{2} \cap l_{\theta_{0}}^{\eta}$, the proof of (3.35) yields also the first step of the induction. Thus, we conclude Lemma 3.2 by proving (3.35) and the boundedness of $E_{y}\left(\tau^{1}\right), T y \in \gamma_{1} \cup l_{\theta_{0}}^{\eta}$. By definition of $\tau^{1}$, both sides of (3.35) are equal to zero if $y \in \gamma_{1}$. Therefore, (3.35) is nontrivial only if $y \in l_{\theta_{0}}^{\eta}$.

In order to obtain a bound on $E_{y}\left(\tau^{1}\right)$, we consider the diffusion process in the covering domain

$$
\hat{\Omega}_{J_{1}}^{\infty}=\left\{J_{1} \leq J<\infty, \infty<\theta<\infty\right\} .
$$

The diffusion on $\Omega_{J_{1}}^{\infty}$ can be extended to $\hat{\Omega}_{J_{1}}^{\infty}$ in a natural way. Define $\bar{\Omega}_{J_{1}}^{\infty} \subset \hat{\Omega}_{J_{1}}^{\infty}$ as

$$
\overline{\hat{\Omega}}_{J_{1}}^{\infty}=\hat{\Omega}_{J_{1}}^{\infty}-\left\{\bigcup_{k \neq 0} l_{\theta_{0}+2 \pi k}^{\eta}\right\}
$$

where $k$ runs over the nonzero integers.

Let $\hat{\tau}_{1}$ be the escape time to $\overline{\hat{\Omega}}_{J_{1}}^{\infty}$, due to the diffusion process in the covering space. By definition of $\tau^{1}$ we obtain $\tau^{1}=\hat{\tau}^{1}$ for any sample path of the diffusion starting at $l_{\theta_{0}}^{\eta}$ in both $\Omega_{J_{1}}^{\infty}$ and $\hat{\Omega}_{J_{1}}^{\infty}$, respectively.

We proceed by defining a barrier function, $V$, on $\bar{\Omega}_{J_{1}}^{\infty}$. If

$$
L^{\varepsilon} V \geq 1
$$


and $V$ is twice differentiable on $\overline{\hat{\Omega}}_{J_{1}}^{\infty}$, then by the Ito formula, for $y \in l_{\theta_{0}}^{\eta}$,

$$
E_{y}\left(\tau^{1}\right)=E_{y}\left(\hat{\tau}^{1}\right) \leq E_{y}\left(V\left(z\left(\tau^{2}\right)\right)\right)-V(y) \leq 2 \sup _{w \in l_{\theta_{0}}^{\eta}} V(w) .
$$

In (3.37) we identified the diffusions on the original and covering domains. On $\Omega_{J_{1}}^{\infty}$, $V$ is not necessarily a single-valued function. We turn now to the definition of $V$.

Define $\Theta(J) \geq 0$ by

$$
\begin{aligned}
& \Theta(J)=0, \quad J_{1} \leq J \leq J_{1}+\eta, \\
& \Theta(J)=1, \quad J_{1}+2 \eta \leq J<\infty, \\
& \Theta \text { smooth and positive on } \eta+J_{1} \leq J<\infty .
\end{aligned}
$$

Let $u=u(J)$ :

$$
L^{\varepsilon} u>1 \text { on } J_{1}<J<J_{1}+2 \eta .
$$

For given $C>0$ and $\varepsilon>0$, define

$$
V=\varepsilon C \Theta(J) \hat{\theta}+(1-\Theta(J)) u(J)
$$

where $\hat{\theta}$ is given by

$$
\begin{aligned}
\hat{\theta}=\theta & \text { on }-2 \pi \leq \theta \leq 2 \pi, \\
\hat{\theta}=\theta \bmod 2 \pi & \text { on }(\theta \geq 2 \pi) \cup(\theta<-2 \pi) .
\end{aligned}
$$

Evidently, $V$ is smooth on $\overline{\hat{\Omega}}_{J_{1}}^{\infty}$, and globally bounded.

Consider $L^{\varepsilon} V$. Since $\bar{\nabla} H \cdot \nabla \equiv \omega(J)(\partial / \partial \theta)$ by definition, a simple computation yields

$$
L^{\varepsilon} V=C \phi \omega+\varepsilon C(\Delta+Q \cdot \nabla) \Theta \hat{\theta}+L^{\varepsilon}\{(1-\Theta) u\}
$$

By the lower bound on $\omega$ and the definition of $\Theta$, the first term above is bounded from below on $J \geq J_{1}+\eta+\delta$, for any $\delta>0$. The last term of (3.38) exceeds 1 on an interval $\left(J_{1}, J_{1}+\eta+\delta\right)$ for $\delta>0$ small enough, by the definition of $u$. The middle term is uniformly bounded on $\Omega_{J_{1}}^{\infty}$ by (1.10), (1.11) and (1.13). Thus we may choose $\delta>0$ small enough, then $\varepsilon>0$ small enough to obtain (3.36).

In addition to the above bound, we need a lower bound

$$
\inf _{w=l_{\theta_{0}}^{\eta}} E_{w}\left(\tau^{1}\right) \geq D \varepsilon
$$

for some $D>0$, independent of $\varepsilon$.

Consider the deterministic system obtained by removing the white noise component from the diffusion process

$$
\dot{\bar{z}}^{0}=\varepsilon^{-1} \bar{\nabla} H\left(\bar{z}^{0}\right)+Q\left(\bar{z}^{0}\right), \quad \bar{z}^{0}(0)=w \in l_{\mathscr{O}_{0}}^{\eta} .
$$

Since $Q$ increases at most linearly in $|z|$ by (1.10) we obtain by (1.13) the uniform bound on $Q \cdot \nabla_{z} \theta$. From (3.40), in terms of $(J, \theta)$

$$
\frac{d}{d t} \theta\left(\bar{z}^{0}(t)\right)=\frac{1}{\varepsilon} \omega\left(\bar{z}^{0}(t)\right)+Q \cdot \nabla_{z} \theta
$$

Since $\omega$ is bounded from below and $Q \cdot \nabla_{z} \theta$ is uniformly bounded, we observe that for $\varepsilon$ small enough, the first hitting time of $l_{\theta_{0}}^{\eta}$ due to the deterministic process (3.40) is bounded from below uniformly on $w \in l_{\theta_{0}}^{\eta}$ provided $\varepsilon$ is small enough. On 
the other hand, comparing (3.40) to the original diffusion process with given $\varepsilon>0$ we obtain

$$
P_{w}\left(\sup _{0 \leq s \leq \varepsilon T}\left|z^{\varepsilon}(s)-\bar{z}^{0}(s)\right| \geq \nu\right) \leq C(\nu, T) \varepsilon^{2} .
$$

(See [2, Chapter 2].) Since $Q$ and $\bar{\nabla} H$ are both uniformly Lipschitz, the constant $C(\nu, t)$ above is independent of the choice of $w \in l_{\theta_{0}}^{\eta}$. From the above remark, we may define a constant $T>0$ for which

$$
\sup _{0 \leq t \leq \varepsilon T}\left|\theta\left(\bar{z}^{0}(t)\right)\right|<2 \pi-\nu, \quad \forall \bar{z}^{0}(0)=w \in l_{\theta_{0}}^{\eta} .
$$

Using (3.41) we conclude

$$
P_{w}\left(\tau^{1} \geq \varepsilon T\right) \geq 1-C(\nu / 2, T) \varepsilon^{2}, \quad \forall w \in l_{\theta_{0}}^{\eta}
$$

and (3.39) follows. We complete the proof of Lemma 3.2 by proving (3.35) in Appendix B.

Appendix A. The uniform bound on (3.20) is an immediate consequence from the assumptions on $J$ and $Q(1.10,11)$ and $(1.6)$. Taking the $z$-derivative of the first term on the r.h.s. of (3.20) and using (3.21), we obtain

$$
\nabla_{z} \frac{|\nabla J|^{2}}{\left\langle|\nabla J|^{2}\right\rangle}=\frac{\nabla_{z}|\nabla J|^{2}}{\left\langle|\nabla J|^{2}\right\rangle}-\frac{1}{\left\langle|\nabla J|^{2}\right\rangle^{2}}|\nabla J|^{2}\langle\Delta J\rangle \nabla J
$$

The r.h.s. of (A.1) is estimated by $O\left(|z|^{-1}\right)$, using (1.11) and (1.12).

Next, consider

$$
e^{-\chi(J)} \int_{J}^{\infty} e^{\chi(s)} d s=-\left[\frac{d}{d J} \chi\right]^{-1}+\beta(J)
$$

where

$$
\beta(J) \equiv e^{-\chi(J)} \int_{J}^{\infty} e^{\chi(s)} \frac{d}{d J}\left(\left[\frac{d}{d J} \chi\right]^{-1}\right)(s) d s .
$$

By definition and our assumptions

$$
\frac{d \chi}{d J}=\frac{\langle Q \cdot \nabla J\rangle}{\left\langle|\nabla J|^{2}\right\rangle}=O(1) .
$$

Using Green's equality (3.21), we obtain

$$
\frac{d^{2} \chi}{d J^{2}}=\frac{\langle\operatorname{div} \cdot Q\rangle}{\left\langle|\nabla J|^{2}\right\rangle}+\frac{\langle Q \cdot \nabla J\rangle\langle\Delta J\rangle}{\left\langle|\nabla J|^{2}\right\rangle^{2}}
$$

and, by the assumptions of the theorem

$$
\frac{d^{2} \chi}{d J^{2}}(z)=O\left(|z|^{-2}\right) .
$$

Hence

$$
\left|\nabla_{z} \frac{d}{d J} \chi\right|=\left|\nabla J \frac{d^{2}}{d J^{2}} \chi\right|=O\left(|z|^{-1}\right)
$$


Since $|d \chi / d J|$ is bounded from below for $|z|$ large enough (see 1.6), we obtain a uniform bound on the first term on the r.h.s. of (A.2). By (A.5) and (1.6), $\beta(J)=O\left(|z|^{-2}\right)$. By (A.6) and the lower bound on $|d \chi / d J|$ we also obtain

$$
\nabla_{z}[d \chi / d J]^{-1}=O\left(|z|^{-1}\right) .
$$

As for $\beta(J)$

$$
\nabla_{z} \beta=\left\{\beta \frac{d \chi}{d J}-\frac{d}{d J}\left[\frac{d}{d J} \chi\right]^{-1}\right\} \nabla_{z} J .
$$

Using the $O\left(|z|^{-1}\right.$ ) estimate on $\beta$, (A.5) and (1.11), we get

$$
\left|\nabla_{z} \beta\right|=O\left(|z|^{-1}\right) .
$$

Hence

$$
\nabla_{z}\left[e^{-\chi(s)} \int_{J}^{\infty} e^{\chi(s)} d s\right]=O\left(|z|^{-1}\right) .
$$

Finally, we have to estimate the term in curly brackets of (3.20):

$$
\left|\nabla_{z}\{\cdot\}\right|=O\left(|z|^{-1}\right) .
$$

(A.11) is easily verified by using (3.21) together with (1.11), (1.12) and (1.10).

Appendix B. We split $y \in l_{\theta_{0}}^{n}$ into two cases.

Case (a). $y \in l_{\theta_{0}}^{\eta}, J(y) \leq J_{1}+2 \eta$. Then

$$
\begin{aligned}
& E_{y}\left(\int_{0}^{\tau^{1}} \tilde{\xi}(z(s)) d s\right)=E_{y}\left(\int_{0}^{\tau^{1}} \tilde{\xi}(z(s)) d s ; \sup _{0<t \leq \tau^{1}} J(z(t))<J_{1}+4 \eta\right) \\
& \quad+E_{y}\left(\int_{0}^{\tau^{1}} \tilde{\xi}(z(s)) d s ; \exists 0<t \leq \tau^{1}, J(z(t))=J_{1}+4 \eta\right) .
\end{aligned}
$$

By definition of $\tilde{\xi}$ (see (3.29)), we estimate the first term in (B.1) by $\delta E_{y}\left(\tau^{1}\right) / 2$. As for the second term, consider

$$
\begin{aligned}
P_{y}(\exists 0 & \left.<t \leq \tau^{1}, J(z(t))=J_{1}+4 \eta\right) \\
& \leq \sup _{\left\{\omega ; J(w)=J_{1}+3 \eta\right\}} P_{w}\left(\exists 0<t \leq \tau^{1}, J(z(t))=J_{1}+4 \eta\right) \\
& \leq \sup _{\left\{\omega ; J(w)=J_{1}+3 \eta\right\}} P_{w}\left(\sup _{0 \leq s \leq \varepsilon T}\left|z(s)-\bar{z}^{0}(s)\right| \geq \frac{\eta}{2}\right) \leq C(\eta, T) \varepsilon^{2}
\end{aligned}
$$

by (3.41), provided $\varepsilon T$ is chosen so that the orbit of (3.40) completes at least one revolution at this time. By (B.2) and the Cauchy-Schwarz inequality, we estimate the second term of (B.1) by

$$
\begin{aligned}
M\left[E_{y}\left(\tau^{1}\right)^{2}\right]^{1 / 2}\left[C(\eta, T) \varepsilon^{2}\right]^{1 / 2} & \leq C M \sup _{z \in \Omega_{J_{1}}^{\infty}}\left[E_{z}\left(\tau^{1}\right)\right]^{1 / 2}\left[E_{y}\left(\tau^{1}\right)\right]^{1 / 2} \varepsilon \\
& \leq C(\eta)\left[E_{y}\left(\tau^{1}\right)\right]^{1 / 2} \varepsilon
\end{aligned}
$$

In (B.3) we used the inequality

$$
E_{y}\left(|\tau|^{2}\right) \leq \sup _{z \in \Omega} E_{z}(\tau) \cdot E_{y}(\tau)
$$


where $\tau$ is the escape time from an arbitrary domain $\Omega$. The r.h.s. of (B.3) is now estimated by

$$
C(\eta)\left[E_{y}\left(\tau^{1}\right)\right]^{1 / 2} \varepsilon \leq C(\eta) D^{-1 / 2} \varepsilon^{1 / 2} E_{y}\left(\tau^{1}\right) \leq \delta E_{y}\left(\tau^{1}\right) / 2
$$

using (3.39), provided $\varepsilon$ is small enough. This completes the proof of Case (a).

Case (b). $y \in l_{\theta_{0}}^{\eta}, J(y)>J_{1}+2 \eta$. Let $T_{y}^{\varepsilon}$ be the hitting time of $l_{\theta_{0}+2 \pi}^{\eta}$ due to the deterministic system $(3.40), \bar{z}_{0}(0)=y$. By the assumptions of the theorem, we obtain a uniform bound

$$
\sup _{y \in l_{\theta_{0}}^{\eta}} T_{y}^{\varepsilon}<\varepsilon \bar{T}
$$

In fact, the frequency $\omega(J)$ is bounded from below and $Q \cdot \nabla_{z} \theta$ is uniformly bounded by $(1.10),(1.13)$. Hence

$$
\frac{d}{d t} \theta\left(\bar{z}^{0}(t)\right) \geq \varepsilon^{-1} \inf _{z} \omega-\sup _{z}\left|Q \cdot \nabla_{z} \theta\right| \geq \varepsilon^{-1} C
$$

and (B.5) follows upon inserting $\bar{T}=2 \pi / C$.

On the space of orbits starting at $y$ we define the event

$$
\mathscr{M}^{\varepsilon}=\left\{z ; \sup _{0 \leq s \leq 2 \varepsilon \bar{T}}\left|z(s)-\bar{z}^{0}(s)\right| \leq \frac{\delta}{4 \sup _{z}|\nabla \tilde{\xi}|+N}\right\}
$$

where $N>4$ will be determined later on.

By (3.39) with $\nu=\delta / N$ we obtain

$$
P_{y}\left(\mathscr{M}^{\varepsilon}\right) \geq 1-C(\delta / N) \varepsilon^{2} .
$$

An analysis similar to (B.2)-(B.4) yields

$$
E_{y}\left(\int_{0}^{\tau^{1}} \tilde{\xi}(z(s)), \sim \mathscr{M}^{\varepsilon}\right) \leq M\left[C\left(\frac{\delta}{N}\right)\right]^{1 / 2}\left[E_{y}\left(\tau^{1}\right)\right]^{1 / 2} \varepsilon \leq \frac{\delta}{3} E_{y}\left(\tau^{1}\right),
$$

provided $\varepsilon$ is small enough. We estimate now

$$
\begin{aligned}
E_{y}\left(\int_{0}^{\tau^{1}} \xi(z(s)) d s, \mathscr{M}^{\varepsilon}\right)= & E_{y}\left(\int_{0}^{\tau^{1}}\left(\tilde{\xi}(z(s))-\tilde{\xi}\left(\bar{z}_{0}(s)\right)\right) d s, \mathscr{M}^{\varepsilon}\right) \\
& +E_{y}\left(\int_{0}^{\tau^{1}} \tilde{\xi}\left(\bar{z}^{0}(s)\right) d s, \mathscr{M}^{\varepsilon}\right) .
\end{aligned}
$$

The first term on the right of (B.7) is estimated by

$$
\sup |\nabla \tilde{\xi}| E_{y}\left(\int_{0}^{\tau^{1}}\left|z(s)-\bar{z}^{0}(0)\right| d s, \mathscr{M}^{\varepsilon}\right) \leq \frac{\delta}{4} E_{y}\left(\tau^{1}\right) .
$$

In fact, the definitions of $\mathscr{M}^{\varepsilon}$ and $\tau^{1}$ together with the special choice of $y$ due to Case (b), yield for $\varepsilon$ small enough:

$$
\sup _{\mathscr{M}} \tau^{1} \leq 2 \varepsilon \bar{T}
$$


so the integral in (B.8) is bounded by $\delta /(4 \sup |\nabla \tilde{\xi}|)$, by definition. The second term in (B.7) is written as

$$
E_{y}\left(\int_{0}^{\tau_{1}}\left(\tilde{\xi}\left(\bar{z}^{0}(s)\right)-\tilde{\xi}\left(z^{0}(s)\right) d s, \mathscr{M}_{\varepsilon}\right)+E_{y}\left(\int_{0}^{\tau_{1}} \tilde{\xi}\left(z^{0}(s)\right) d s, \mathscr{M}_{\varepsilon}\right) .\right.
$$

where $z^{0}(s)$ is the orbit of the unperturbed system

$$
\dot{z}^{0}=\frac{1}{\varepsilon} \bar{\nabla} H\left(z^{0}\right), \quad z^{0}(0)=y .
$$

To estimate the first term in (B.10) we have to compare the deviation of $z^{0}(s)$ from $\bar{z}^{0}(s)$ on a $2 \varepsilon \bar{T}$ time interval. Using the assumptions of the theorem (in particular, the uniform Lipschitz conditions on $Q$ and $\bar{\nabla} H$ ), we obtain

$$
\sup _{0 \leq s \leq 2 \varepsilon \bar{T}}\left|z^{0}(s)-\bar{z}^{0}(s)\right| \leq \varepsilon C|y|, \quad z^{0}(0)=y,
$$

for $\varepsilon$ and $|y|$ independent of $C$. Taking into account the estimate on $|\nabla \tilde{\xi}|$ due to Lemma 3.1, we obtain

$$
\begin{aligned}
& E_{y}\left(\int_{0}^{\tau_{1}} \tilde{\xi}\left(\bar{z}^{0}(s)\right)-\tilde{\xi}(z(s)) d s, \mathscr{M}_{\varepsilon}\right) \\
& \quad \leq \sup _{|z| \geq|y| / 2}|\nabla \tilde{\xi}(z)| \sup _{0 \leq s \leq 2 \varepsilon \tilde{T}}\left|z^{0}(s)-\bar{z}^{0}(s)\right| E_{y} \tau^{1} \\
& \quad \leq \varepsilon C^{\prime} E_{y} \tau^{1} \leq \frac{\delta}{4} E_{y} \tau^{1}, \quad \forall y \in l_{\theta_{0}}^{\eta} .
\end{aligned}
$$

The second term in (B.10) is written as

$$
\int_{0}^{T_{y}^{0}} \tilde{\xi}\left(z^{0}(s)\right) d s+E_{y}\left(\int_{T_{y}^{0}}^{\tau^{1}} \tilde{\xi}\left(z^{0}(s)\right) d s, \mathscr{M}^{\varepsilon}\right)
$$

where $T_{y}^{0}$ is the period of (B.11). The first term in (B.13) is identically zero by the definition of $\tilde{\xi}$. The second term in is estimated by

$$
\sup _{\Omega_{J_{1}}^{\infty}}|\tilde{\xi}| \sup _{\mathscr{K}^{\varepsilon}}\left|\tau^{1}-T_{y}^{0}\right| \leq \sup _{\Omega_{J_{1}}^{\infty}}|\tilde{\xi}|\left(\sup _{\mathscr{K}^{\varepsilon}}\left|\tau^{1}-T_{y}^{\varepsilon}\right|+\left|T_{y}^{\varepsilon}-T_{y}^{0}\right|\right)
$$

where $T_{y}^{\varepsilon}$ is, as before, the hitting time of $l_{\theta_{0}}^{\eta}$ due to (3.40). By the definition of $\tau^{1}$ and $\mathscr{M}^{\varepsilon}, \sup _{\mathscr{M}^{\varepsilon}}\left|\tau^{1}-T_{y}^{\varepsilon}\right|$ is estimated by the time in which the orbits of (3.40) cross a $\delta / N$ neighborhood of $l_{\theta_{0}}^{\eta}$. This time is estimated by $\varepsilon O(\delta / N)$ uniformly in $y \in l_{\theta_{0}}^{\eta}$. Taking $N$ large, we bound this time by

$$
\varepsilon O\left(\frac{\delta}{N}\right)<\frac{1}{4} \frac{D}{\sup |\tilde{\xi}|} \delta
$$

where $D$ is defined in (3.39). Hence:

$$
\sup |\tilde{\xi}| \sup _{\mathscr{M}^{\varepsilon}}\left|\tau^{1}-T_{y}^{\varepsilon}\right| \leq \frac{\delta}{4} E_{y}\left(\tau^{1}\right) .
$$

To obtain a bound on $\left|T_{y}^{\varepsilon}-T_{y}^{0}\right|$, we use the assumption on $H$ and $Q$. It is easy to see that $\left|T_{y}^{\varepsilon}-T_{y}^{0}\right|$ is estimated by $O\left(\varepsilon^{2}\right)$, uniformly in $y \in l_{\theta_{0}}^{\eta}$. Hence for $\varepsilon$ small enough

$$
\sup |\tilde{\xi}|\left|T_{y}^{\varepsilon}-T_{y}^{0}\right| \leq \frac{\delta}{4} D \varepsilon \leq \frac{\delta}{4} E_{y}\left(\tau^{1}\right), \quad \forall y \in l_{\theta_{0}}^{\eta}
$$


Combining (B.6) with (B.8), (B.12) and (B.16) we complete the desired estimate on $E_{y}\left(\int_{0}^{\tau_{1}} \tilde{\xi}\left(z^{\varepsilon}(s)\right)\right)$.

\section{REFERENCES}

1. R. N. Bhattacharya, Criteria for recurrence and existence of invariant measures for multidimensional diffusions, Ann. Probab. 6 (1978), 541-553.

2. M. Freidlin, and A. Wentzell, Random perturbations of dynamical systems, Springer-Verlag, 1984.

3. A. Friedman, Stochastic differential equations and application, Vol. I, Academic Press, 1976.

4. R. Z. Khas'minskii, Ergodic properties of recurrent diffusion process and stabilization of the solution of the Cauchy problem for parabolic equations, Theory Probab. Appl. 5 (1960), 179191.

5. G. Wolansky, Stochastic perturbations to conservative dynamical systems on the plane. I: Convergence of invariant distributions, Trans. Amer. Math. Soc. 309 (1988), 621-639.

Department of Theoretical Mathematics, The Weizmann Institute of SciENCE, REHOVOT 76100, ISRAEL 